\title{
Temperature Coefficient of the Refractive Index of Diamond-and Zinc-Blende-Type Semiconductors
}

Peter Y. Yu and Manuel Cardona

Brown University, Providence, Rhode Island and Deutsches Elektronen-Synchrotron DESY, Hamburg, Germany (Received 11 May 1970)

We have calculated the temperature coefficient of the long-wavelength refractive index of several group-IV and III-V semiconductors, using the Penn model for the electronic contribution to the dielectric constant. The isotropic band gap of this model is identified with the band gap at the $X$ point of the Brillouin zone, which can be simply expressed in terms of pseudopotential coefficients. The explicit temperature dependence of this gap is calculated by applying to these pseudopotential coefficients the appropriate Debye-Waller factors. The thermal expansion effect is obtained in the manner suggested recently by Van Vechten. Good agreement between the calculated and the observed temperature dependence of the long-wavelength refractive index is found.

\section{INTRODUCTION}

Recently, Phillips's theory of ionicity ${ }^{1}$ has been applied successfully to calculate various properties, namely, ionization potential, ${ }^{2}$ cohesive energy, ${ }^{3}$ nonlinear optical susceptibilities, ${ }^{4-6}$ etc., of a large number of covalent and ionic compounds. At the heart of this theory is a very simple isotropic one-gap model of the band structure due to Penn. ${ }^{7}$ Heine and Jones ${ }^{8}$ pointed out that one reason why such a simple model works for the diamond-type semiconductors is that the gap on the surface of the Jones zone is nearly constant over a large area. By identifying the "Penn gap" with the band gap at the center of the (110) Jones zone face, they calculated the hydrostatic pressure coefficient of the dielectric constant of $\mathrm{Si}$ and Ge. In this paper, we generalize Heine and Jones's calculation to the III-V semiconductors and obtain the "Penn gap" as a function of the pseudopotential coefficients. We then calculate with this model the temperature coefficient of the refractive index of several group-IV and group-III-V semiconductors.

\section{PENN MODEL}

The electronic contribution to the long-wavelength dielectric constant of a solid in the Penn model is given by ${ }^{7,9}$

$$
\epsilon(0) \cong 1+D\left(\omega_{p}^{2} / \omega_{g}^{2}\right),
$$

where $\omega_{p}$ is the plasma frequency of the valence electrons, $\omega_{g}$ is the Penn gap, and $D$ is a parameter introduced by Van Vechten ${ }^{9}$ to take into account the effect of $d$-like core electrons. It is difficult to speculate on how $D$ will vary with temperature and volume. Since $D$ is of the order of unity and its variations are not expected to be large, we shall neglect this factor in our calculation.

By differentiation of Eq. (1) with respect to tem- perature (throughout this paper, pressure is assumed to be constant when the $d$ sign of differentiation is used), we obtain

$$
\begin{aligned}
\frac{1}{\epsilon} \frac{d \epsilon}{d T} & =\frac{\epsilon-1}{\epsilon}\left[\frac{1}{\omega_{p}^{2}} \frac{d \omega_{p}^{2}}{d T}-2\left(\frac{1}{\omega_{g}} \frac{d \omega_{g}}{d T}\right)\right] \\
& =\frac{\epsilon-1}{\epsilon}\left[-3 \alpha-2\left(\frac{1}{\omega_{g}} \frac{d \omega_{g}}{d T}\right)\right],
\end{aligned}
$$

where $\alpha$ is the coefficient of linear expansion. In Eq. (2) we have made use of the known proportionality of $\omega_{p}^{2}$ to the valence electron concentration. We decompose the temperature coefficient of $\omega_{g}$ which appears in Eq. (2) into its "explicit" temperature dependence at constant volume and its volume expansion effect:

$$
\frac{1}{\omega_{g}} \frac{d \omega_{g}}{d T}=\frac{1}{\omega_{g}}\left(\frac{\partial \omega_{g}}{\partial T}\right)_{V}+3 \alpha \frac{V}{\omega_{g}}\left(\frac{\partial \omega_{g}}{\partial V}\right)_{T} .
$$

\section{EXPLICIT TEMPERATURE DEPENDENCE OF PENN GAP}

Following Heine and Jones, ${ }^{8}$ we identify the Penn gap $\omega_{g}$ with the gap at the $X$ point of the Brillouin zone. As is well known, its energy is close to that of the strongest peak in the oscillator strength and the reflectivity of the group-IV and group-III-V semiconductors. ${ }^{10}$ An expression for this gap as a function of the pseudopotential form factors $v(111)$ and $v(220)$ has been calculated by Heine and $\mathrm{Jones}^{8}$ for germanium-type materials using perturbation theory. This expression can be easily generalized to zinc-blende-type compounds. We find, in rydbergs, ${ }^{11}$

$$
\omega_{g}^{ \pm}=2 v_{s}(220) \pm 2 v_{a}(200)+\frac{2\left[v_{s}(111) \pm v_{a}(111)\right]^{2}}{(2 \pi / a)^{2}},
$$

where $a$ is the lattice constant in Bohr radii. The subscripts $s$ and $a$ of the pseudopotential coefficients 
TABLE I. Various parameters used for calculating the temperature dependence of the long-wavelength refractive index of group-IV and III-V semiconductors.

\begin{tabular}{|c|c|c|c|c|c|c|c|}
\hline & $v_{s}(220)$ & $\begin{array}{c}\text { Pseudopotential } \\
\text { coeff. (Ry) } \\
v_{s}(111) \\
\end{array}$ & $v_{a}(111)$ & $\epsilon(0)^{b}$ & $\begin{array}{c}\text { Coeff. of linear } \\
\text { expansion at } \\
\text { room temperature } \\
\left({ }^{\circ} \mathrm{K}^{-1}\right)\end{array}$ & $\begin{array}{c}\text { Debye temp. } \\
\Theta_{D} \text { at } \\
300^{\circ} \mathrm{K} \\
\left({ }^{\circ} \mathrm{K}\right) \\
\end{array}$ & $\begin{array}{l}\text { Lattice constant } \\
\text { (in Bohr radii) }^{\mathrm{b}}\end{array}$ \\
\hline $\mathrm{C}$ & $\begin{array}{l}-0.022 \\
+0.337\end{array}$ & $\begin{array}{l}-0.514 \\
-0.811\end{array}$ & $\cdots$ & 5.7 & $1.1 \times 10^{-6 \mathrm{c}}$ & $1880^{d}$ & 6.74 \\
\hline $\mathrm{Si}$ & +0.04 & -0.21 & $\cdots$ & 12.0 & $2.5 \times 10^{-6^{c}}$ & $647^{e}$ & 10.26 \\
\hline $\mathrm{Ge}$ & +0.01 & -0.23 & $\cdots$ & 16.0 & $5.7 \times 10^{-6^{f}}$ & $354^{\mathrm{e}}$ & 10.69 \\
\hline GaAs & +0.01 & -0.23 & +0.07 & 10.9 & $5.7 \times 10^{-6^{\mathrm{f}}}$ & $360^{f}$ & 10.68 \\
\hline $\mathrm{GaSb}$ & 0.0 & -0.22 & +0.06 & 14.4 & $6.3 \times 10^{-6^{\mathrm{f}}}$ & $230^{f}$ & 11.56 \\
\hline InAs & 0.0 & -0.22 & +0.08 & 12.3 & $5.4 \times 10^{-68}$ & $270^{f}$ & 11.41 \\
\hline $\mathrm{InSb}$ & 0.0 & -0.20 & +0.06 & 15.7 & $5.1 \times 10^{-6^{f}}$ & $150^{f}$ & 12.24 \\
\hline In $P$ & +0.01 & -0.23 & +0.07 & 9.6 & $4.9 \times 10^{-6 \mathrm{~g}}$ & $420^{f}$ & 11.09 \\
\hline $\mathrm{GaP}$ & +0.03 & -0.22 & +0.12 & 9.1 & $\begin{array}{l}5.81 \times 10^{-6} \mathrm{~h} \\
5.3 \times 10^{-6} \mathrm{~g}\end{array}$ & $470^{\mathrm{i}}$ & 10.3 \\
\hline
\end{tabular}

${ }^{\mathrm{a}} \mathrm{M}$. L. Cohen and T. K. Bergstresser, Phys. Rev. 141, 789 (1966); L. R. Saravia and D. Brust, ibid. 170, 683 (1968).

${ }^{\mathrm{b}}$ See J. A. Van Vechten, Phys. Rev. 182, 891 (1969), and references therein.

${ }^{\mathrm{c}}$ American Institute of Physics Handbook (McGraw Hill, New York, 1963), 2nd ed., pp. 4-66.

${ }^{d} J$. E. Desnoyers and J. A. Morrison, Phil. Mag. 3, 42 (1958).

${ }^{e} \mathrm{P}$. Flubacher, A. J. Leadbetter, and J. A. Morrison, Phil. Mag. 4, 273 (1959).

${ }^{\mathrm{f} S e m i c o n d u c t o r s}$ and Semimetals, edited by R.K. Willardson and A. C. Beer (Academic Press, New York, 1966), Vol. 2 .

${ }^{\mathrm{g}} \mathrm{H}$. Welker and H. Weiss, in Solid State Physics, edited by F. Seitz and D. Turnbull (Academic Press, New York, 1956), Vol. 3.

${ }^{\text {h}}$ E. D. Pierron, D. L. Parker, and J. B. Mc Neely, J. Appl. Phys. 38, 4669 (1967).

${ }^{\mathbf{i}}$ The Debye temperature of GaP at room temperature is not available. The value given in Table $\mathrm{I}$ is estimated from its $0^{\circ} \mathrm{K}$ Debye temperature $\left(446^{\circ} \mathrm{K}\right)$ calculated from the elastic constants [E. F. Steigmeier, Appl。 Phyś. Letters 3 , 6 (1963)].

$v(220), v(200)$, and $v(111)$ denote their symmetric and antisymmetric components, respectively. The antisymmetric coefficients $v_{a}(200)$ and $\omega_{a}(111)$ are responsible for the splitting of the $X_{1}$ conductiun bands [ \pm sign in Eq. (4)] but does not change, to first order, the average value of $\omega_{g}$. Since the splitting is known to be small for III-V compounds, it can be neglected for the purpose of calculating dielectric properties. We thus obtain

$$
\omega_{g}=2\left(v_{s}(220)+\frac{\left[v_{s}(111)\right]^{2}+\left[v_{a}(111)\right]^{2}}{(2 \pi / a)^{2}}\right) .
$$

The values of the pseudopotential coefficients $v_{s}(220), v_{s}(111)$, and $v_{a}(111)$ for several group-IV and group-III-V semiconductors are listed in Table I together with other parameters relevant for our calculations. The values of $\omega_{g}$ obtained from these parameters with Eq. (5) are listed in Table II, together with the values obtained from the dielectric constant [see Eq. (1)] by Van Vechten ${ }^{9}$ and the energy $E_{2}$ of the corresponding reflectivity peak. The good agreement between these three sets of values confirms the validity of Eq. (5).

Equation (5) can be used to calculate the temperature dependence of $\omega_{g}$ by multiplying the Fourier component of the pseudopotential $v(g)$ by the appropriate Debye-Waller factor ${ }^{12,13}$ :

$$
\begin{aligned}
\exp \left[-B(T)|\overrightarrow{\mathrm{g}}|^{2}\right]= & \exp \left[-\left(|\overrightarrow{\mathrm{g}}|^{2} / 6\right)\right]\left\langle u^{2}\right\rangle \\
& \text { (for cubic crystals). }
\end{aligned}
$$

For a crystal with $r$ atoms per unit cell the average square displacement of atom $K$ is ${ }^{14}$

$$
\left\langle u_{K}^{2}\right\rangle=\frac{1}{2 N M_{K}} \sum_{\vec{a}, j}|\overrightarrow{\mathrm{e}}(K, j, \overrightarrow{\mathrm{q}})|^{2} \frac{\operatorname{coth} \frac{1}{2} \beta \omega_{j}(\overrightarrow{\mathrm{q}})}{\omega_{j}(\overrightarrow{\mathrm{q}})},
$$

where $\beta=k T, M_{K}$ is the mass of the $K$ th atom, $N$ is the number of unit cells per unit volume, $\omega_{j}(\overrightarrow{\mathrm{q}})$ is the frequency of the normal mode with wave vector $\overrightarrow{\mathrm{q}}$ in the $j$ th branch, and $\overrightarrow{\mathrm{e}}(K, j, \overrightarrow{\mathrm{q}})$ is the polarization vector of the corresponding displacement of atom $K$.

In group-IV semiconductors, the two atoms of the unit cell are identical and Eq. (7) simplifies to

$$
\left\langle u^{2}\right\rangle=\frac{1}{2 N M} \sum_{\overrightarrow{\mathrm{a}}, j} \frac{\operatorname{coth} \frac{1}{2} \beta \omega_{j}(\overrightarrow{\mathrm{q}})}{2 \omega_{j}(\overrightarrow{\mathrm{q}})} .
$$

We calculate $\partial B(T) / \partial T$ with the Debye model for the phonon spectrum and obtain with Eq. (8) 


$$
\frac{\partial B(T)}{\partial T}=\frac{3}{2} \frac{1}{M k T^{2}} F\left(\frac{\Theta_{D}}{T}\right)
$$

with

$$
F(x)=\frac{1}{x^{2}}\left(\frac{2}{x} \int_{0}^{x} \frac{y d y}{e^{y}-1}-\frac{x}{e^{x}-1}\right) .
$$

We shall use in our calculation values of the Debye temperature $\theta_{D}$ at room temperature, since most experimental data are obtained around room temperature. The function $F(x)$ has been evaluated by Benson and Gill. ${ }^{15}$

For the III-V compounds, it is no longer possible to relate $\left\langle u^{2}\right\rangle$ directly to the phonon spectrum with Eq. (7) since the masses of the two constituent atoms are not equal. However, for those of the III-V compounds we are considering, we find that the ratio of the heavier mass to the lighter is smaller than 2. In the case of the alkali halides, it was found that at room temperature the average square displacements for the anion and cation differ by less than $10 \%$ even for masses which differ by a factor of $3 .{ }^{16}$ Hence, we assume for the sake of simplicity that $\left\langle u_{\mathrm{III}}^{2}\right\rangle=\left\langle u_{\mathrm{V}}^{2}\right\rangle$. The validity of this assumption cannot be checked until the Debye-Waller factors for the III-V compounds have been determined. However, the results of our calculation seem to support this assumption. Thus for the III-V compounds we use the formula

$$
\frac{\partial B_{\mathrm{III}}}{\partial T}=\frac{\partial B_{\mathrm{V}}}{\partial T} \simeq \frac{\partial \bar{B}}{\partial T}=\frac{3}{k T^{2}\left(M_{\mathrm{III}}+M_{\mathrm{V}}\right)} F\left(\frac{\Theta_{D}}{T}\right) .
$$

Combining Eqs. (5) and (6) we obtain

$$
\begin{aligned}
& \left(\frac{\partial \omega_{g}}{\partial T}\right)_{\mathrm{V}} \cong 2\left[v_{s}(220)\left(-|\overrightarrow{\mathrm{g}}(220)|^{2} \frac{\partial \bar{B}}{\partial T}\right)\right. \\
& \left.+\frac{\left[v_{s}(111)\right]^{2}+\left[v_{a}(111)\right]^{2}}{(2 \pi / a)^{2}}\left(-2|\overrightarrow{\mathrm{g}}(111)|^{2} \frac{\partial \bar{B}}{\partial T}\right)\right] .
\end{aligned}
$$

In order to simplify the evaluation of Eq. (12) we note that for the semiconductors we are considering $v(220)$ is small and $|g(220)|^{2} \simeq 2|g(111)|^{2}$. Equation (12) can thus be approximated by the expression

$$
\frac{1}{\omega_{g}}\left(\frac{\partial \omega_{g}}{\partial T}\right)_{\mathrm{V}} \simeq-2|\overrightarrow{\mathrm{g}}(111)|^{2} \frac{\partial \bar{B}}{\partial T}
$$

\begin{tabular}{|c|c|c|c|c|c|}
\hline & $\begin{array}{c}\omega_{g} \text { (theor) } \\
\text { from } \mathrm{Eq} .(5) \\
(\mathrm{eV})\end{array}$ & $\begin{array}{c}\omega_{g}(\operatorname{expt}) \\
\text { from Eq. (1) } \\
(e V)\end{array}$ & $\begin{array}{l}\text { Highest peak } \\
\text { in reflection } \\
\text { spectrum } \\
\left(E_{2}\right)(\mathrm{eV})\end{array}$ & $\begin{array}{l}\frac{d \omega_{g}}{d T}(\text { calc }) \\
\left(\mathrm{eV} /{ }^{\circ} \mathrm{K}\right)\end{array}$ & $\begin{array}{l}\frac{d E_{2}}{d T}(\text { expt }) \\
\left(\mathrm{eV} /{ }^{\circ} \mathrm{K}\right)\end{array}$ \\
\hline $\mathrm{C}$ & $\begin{array}{r}7.8 \\
29.6\end{array}$ & 13.6 & $12.6^{\mathrm{a}}$ & $\begin{array}{l}-1.17 \times 10^{-4} \\
-4.6 \times 10^{-4}\end{array}$ & $\cdots$ \\
\hline $\mathrm{Si}$ & 4.2 & 4.8 & $4.44^{\mathrm{b}}$ & $-1.9 \times 10^{-4}$ & $\begin{array}{l}-2.2 \times 10^{-4} \mathrm{~b} \\
+0.5 \times 10^{-4} \mathrm{c}\end{array}$ \\
\hline $\mathrm{Ge}$ & 4.4 & 4.3 & $4.49^{\mathrm{b}}$ & $-3.3 \times 10^{-4}$ & $\begin{array}{l}-2.4 \times 10^{-4} \mathrm{~b} \\
-2 \times 10^{-4 c}\end{array}$ \\
\hline GaAs & 4.9 & 5.2 & $5.11^{\mathrm{b}}$ & $-3.5 \times 10^{-4}$ & $\begin{array}{l}-3.6 \times 10^{-4 b} \\
-3.3 \times 10^{-4 d}\end{array}$ \\
\hline $\mathrm{GaSb}$ & 4.8 & 4.1 & $4.35^{b}$ & $-4.7 \times 10^{-4}$ & $\begin{array}{l}-4.1 \times 10^{-4} \mathrm{~b} \\
-6.2 \times 10^{-4} \mathrm{e}\end{array}$ \\
\hline InAs & 4.9 & 4.58 & $4.74^{\mathrm{b}}$ & $-3.8 \times 10^{-4}$ & $-5.6 \times 10^{-4^{b}}$ \\
\hline InSb & 4.5 & 3.74 & $4.23^{b}$ & $-6.9 \times 10^{-4}$ & $\begin{array}{r}-3.6 \times 10^{-4} \mathrm{~b} \\
5.4 \times 10^{-4 e}\end{array}$ \\
\hline InP & 5.2 & 5.2 & $5.0^{f}$ & $-2.3 \times 10^{-4}$ & $\cdots$ \\
\hline $\mathrm{GaP}$ & 5.35 & 5.75 & $5.3^{f}$ & $-1.9 \times 10^{-4}$ & $-4.5 \times 10^{-4^{d}}$ \\
\hline
\end{tabular}

with an error smaller than $10 \%$.

TABLE II. Calculated and experimental values of the Penn gap $\omega_{g}$, energy $E_{2}$ of the highest peak in the reflection spectrum, and temperature coefficient of $\omega_{\boldsymbol{g}}$ (theor) and $E_{2}$ (expt).

${ }^{a}$ W. C. Walker and J. Osantowski, Phys。Rev. 134, A153 (1964)。

${ }^{b}$ R. R. L. Zucca and Y. R. Shen (unpublished). These values were measured at $5{ }^{\circ} \mathrm{K}$.

${ }^{\mathrm{c}} \mathrm{M}$. Cardona and H. S. Sommers, Jr., Phys. Rev. 122, 1382 (1961)。

${ }^{d}$ A. G. Thompson, J. C. Woolley, and M. Rubenstein, Can. J. Phys. 44, 2927 (1966)。

${ }^{\mathrm{e}} \mathrm{F}$. Lukes and E. Schmidt, in Proceedings of the International Conference of the Physics of Semiconductors, Exeter, 1962, (The Institute of Physics and The Physical Society, London, 1962), p. 389.

${ }^{\mathrm{f}} \mathrm{M}$. Cardona, in Semiconductors and Semimetals, edited by R. K. Willardson and A。C. Beer (Academic, New York, 1967), VoI. 3, p. 140. Both are room-temperature values. 


\section{VOLUME DEPENDENCE OF PENN GAP}

The volume coefficient of $\omega_{g}$ can be calculated with Eq. (5) and the known derivatives of $v(\overrightarrow{\mathrm{q}})$ with respect to $|\vec{q}| .^{8}$ Since this volume dependence is only responsible for less than $20 \%$ of the total calculated temperature dependence of $\omega_{g}$, we choose for its evaluation the simpler, although possibly less accurate procedure given by Van Vechten. ${ }^{9}$ We start with the equation

$$
\omega_{g}=\left(\omega_{h}^{2}+C^{2}\right)^{1 / 2}
$$

where $\omega_{h}$ is the homopolar energy gap of the material, and $C$ the heteropolar energy gap. In order to calculate the volume dependence of $\omega_{g}$ it is necessary to know the corresponding dependence of $\omega_{h}\left(\omega_{h} \propto a^{-2.48}\right)$ and $C^{9}$. For simplicity we take $(\partial C / \partial V)_{T} \simeq 0$. Our result does not depend critically on this assumption since $C$ only accounts for a small fraction of $\omega_{g}$, whose volume dependence, in turn, only accounts for a small fraction of the total temperature dependence of $\omega_{g}$. We thus find

$$
\frac{V}{\omega_{g}}\left(\frac{\partial \omega_{g}}{\partial V}\right)_{T} \cong-0.8 \times \frac{\omega_{h}^{2}}{\omega_{g}^{2}} .
$$

\section{TEMPERATURE DEPENDENCE OF LONG-WAVELENGTH REFRACTIVE INDEX}

We have listed in Table I the parameters used for our calculation of $(1 / n)(\partial n / \partial T)_{P}$ based on Eqs. (2), (3), (13), and (15). We have used for the Debye temperature the values obtained from specificheat measurements. Batterman and Chipman ${ }^{17}$ found that the Debye temperatures obtained for $\mathrm{Ge}$ and $\mathrm{Si}$ from $\mathrm{x}$-ray intensity measurements were about $20 \%$ lower than those from specific heat. This fact introduces an uncertainty of about $40 \%$ in our calculation of $\left(1 / \omega_{g}\right)\left(\partial \omega_{g} / \partial T\right)_{V}$. We believe this uncertainty is our main source of error.

In Table II we show the temperature coefficient $d \omega_{g} / d T$ calculated with Eqs. (13) and (15) and those determined experimentally for $E_{2}$. The agreement is satisfactory.

In Table III we show the calculated value of $(1 / n)$ $\times(d n / d T)$ at $300{ }^{\circ} \mathrm{K}$ compared with experimental results. Theoretical I are the values obtained by using in Eq. (2) the calculated coefficients $\left(1 / \omega_{g}\right)$ $\times\left(d \omega_{g} / d T\right)$ while Theoretical II are the values obtained by using the experimental $\left(1 / E_{2}\right)\left(d E_{2} / d T\right)$ and assuming $\left(1 / \omega_{g}\right)\left(d \omega_{g} / d T\right)=\left(1 / E_{2}\right)\left(d E_{2} / d T\right)$. We note that the trend in the experimental values in going from one material to another is better represented by Theoretical I than by Theoretical II. A possible explanation is that the values of $d E_{2} / d T$ determined by the wavelength modulation technique are sensitive to the detailed critical point structures while $\omega_{g}$ represents the somewhat different
TABLE III. Temperature coefficient of long-wavelength refractive index of group-IV and III-V semicon-

\begin{tabular}{|c|c|c|c|}
\hline & $\begin{array}{l}\frac{1}{n} \frac{d n}{d T} \\
\text { (theor I) } \\
\left({ }^{\circ} \mathrm{K}^{-1}\right)\end{array}$ & $\begin{array}{c}\frac{1}{n} \frac{d n}{d T} \\
\text { (theore } \Pi) \\
\left({ }^{\circ} \mathrm{K}^{-1}\right)\end{array}$ & $\begin{array}{l}\frac{1}{n} \frac{d n}{d T} \\
\text { (expt) } \\
\left({ }^{\circ} \mathrm{K}^{-1}\right) \\
\end{array}$ \\
\hline $\mathrm{C}$ & 1. $1 \times 10^{-5}$ & $\cdots$ & $0.5 \times 10^{-5} \mathrm{a}$ \\
\hline $\mathrm{Si}$ & $3.7 \times 10^{-5}$ & $4.2 \times 10^{-5}$ & $\begin{array}{l}3.9 \times 10^{-5 b} \\
4.7 \times 10^{-5 c}\end{array}$ \\
\hline $\mathrm{Ge}$ & $6.1 \times 10^{-5}$ & $4.2 \times 10^{-5}$ & $\begin{array}{r}6.9 \times 10^{-5 \mathrm{~d}} \\
10 \times 10^{-5} \mathrm{e}\end{array}$ \\
\hline GaAs & $5.4 \times 10^{-5}$ & $\begin{array}{l}5.7 \times 10^{-5} \\
5.1 \times 10^{-5}\end{array}$ & $4.5 \times 10^{-5 d}$ \\
\hline GaSb & $9.3 \times 10^{-5}$ & $\begin{array}{r}8.0 \times 10^{-5} \\
12.4 \times 10^{-5}\end{array}$ & $8.2 \times 10^{-5^{d}}$ \\
\hline InAs & $6.7 \times 10^{-5}$ & $10.3 \times 10^{-5}$ & $\cdots$ \\
\hline InSb & $15.0 \times 10^{-5}$ & $\begin{array}{r}7.4 \times 10^{-5} \\
11.9 \times 10^{-5}\end{array}$ & $11.9 \times 10^{-5 d}$ \\
\hline InP & $3.6 \times 10^{-5}$ & $\cdots$ & $2.7 \times 10^{-5^{d}}$ \\
\hline GaP & $2.2 \times 10^{-5}$ & $6.8 \times 10^{-5}$ & $3.7 \times 10^{-5^{1}}$ \\
\hline
\end{tabular}
ductors.

${ }^{\mathrm{a} P}$. T. Narasimhan, Proc. Phys. Soc. (London) B68, 315 (1955).

${ }^{\mathrm{b}} \mathrm{M}$. Cardona, W. Paul, and H. Brooks, J. Phys. Chem. Solids 8,204 (1959).

${ }^{\mathrm{c}} \mathrm{F}$. Lukeš , Czech. J. Phys. B10, 317 (1960).

${ }^{\mathrm{d}} \mathrm{M}$. Cardona, in Proceedings of the International Conference on Semiconductor Physics, Prague, 1960 (Academic, New York, 1961) p. 388.

${ }^{\mathbf{e}} \mathrm{F}$. Lukeš, Czech. J. Phys. B10, 745 (1960).

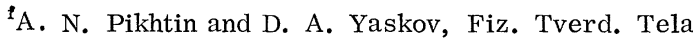
9, 145 (1967) [Soviet Phys. Solid State 9, 107 (1967)].

average gap. The agreement between the calculated values of $(1 / n)(d n / d T)$ and the experimental values is always better than $40 \%$, which is the error of our calculation due to uncertainties in the Debye temperature.

After this work had been completed, it was brought to our attention that Phillips ${ }^{18}$ had also generalized the Heine-Jones model to the zinc-blendetype semiconductors. He obtained for $\omega_{g}$

$$
\begin{aligned}
\omega_{g}^{2}= & \left(2 v_{s}(220)+2 \frac{\left[v_{s}(111)\right]^{2}+\left[v_{a}(111)\right]^{2}}{(2 \pi / a)^{2}}\right)^{2} \\
& +\left(\frac{4 v_{s}(111) v_{a}(111)}{(2 \pi / a)^{2}}\right)^{2}
\end{aligned}
$$

While Eq. (16) has the form of Eq. (14) and gives good agreement with the experiment for the values of $C$, Eq. (5) agrees better with the experimental values of $\omega_{g}$ for the semiconductors considered in this work. In any case our results for $(1 / n)$ $(d n / d T)$ remain the same, since we still obtain Eq. (13), even if Eq. (16) is used for $\omega_{g}$. 


\section{ACKNOWLEDGMENTS}

We wish to express our gratitude to the members of group F 41 of DESY for their hospitality and to R. R. I. Zucca and Y. R. Shen for making available to us their results prior to publication. On of us
(P.Y.Y.) is indebted to General Telephone and Electronics for a Fellowship. We are also grateful to Dr. J. C. Phillips and Dr. J. A. Van Vechten for valuable discussions and for sending us preprints of their work.
${ }^{1}$ J. C. Phillips, Phys. Rev. Letters 20, 550 (1968); Covalent Bonding in Crystals, Molecules, and Polymers (University of Chicago Press, Chicago, 1969).

${ }^{2} \mathrm{~J}$. C. Phillips and J. A. Van Vechten, Phys. Rev. Letters 22, 705 (1969).

${ }^{3}$ J. C. Phillips, Phys. Rev. Letters 22, 645 (1969).

${ }^{4}$ J. C. Phillips and J. A. Van Vechten, Phys. Rev. 183, 709 (1969).

${ }^{5}$ B. F. Levine, Phys. Rev. Letters 22,787 (1969).

${ }^{6} \mathrm{~J}$. A. Van Vechten, M. Cardona, D. E. Aspnes, and R. M. Martin, Proceedings of the International Conference of the Physics of Semiconductors, Cambridge, Mass., 1970 (unpublished).

${ }^{7}$ D. Penn, Phys. Rev. 128, 2093 (1962).

${ }^{8}$ V. Heine and R. O. Jones, J. Phys. C 2, 719 (1969).

${ }^{9}$ J. A. Van Vechten, Phys. Rev. 182, 891 (1969).

${ }^{10}$ M. Cardona, J. Appl. Phys. 36, 2181 (1965).

${ }^{11}$ In Ref. 8 Heine and Jones calculated the band gap at $X$ both by perturbation theory and also more exactly by diagonalisation of a $6 \times 6$ matrix. They found that the perturbation theory gave better agreement with experiment than the more exact method. We found that this is also true for the III-V compounds. As a result we have used the expressions obtained by perturbation theory.

${ }^{12}$ C. Keffer, T. M. Hayes, and A. Bienenstock, Phys. Rev. Letters 21, 1676 (1968).

${ }^{13}$ J. P. Walter, R. R. L. Zucca, M. L。 Cohen, and Y. R. Shen (unpublished).

${ }^{14}$ A. A. Maradudin, E. W. Montroll, and G. H. Weiss, in Solid State Physics, edited by F. Seitz and D. Turnbull (Academic Press, New York, 1963), Suppl. 3, p. 237.

${ }^{15}$ G. C. Benson and E. K. Gill, Can. J. Phys. 44, 614 (1966).

${ }^{16} \mathrm{~W}$. J. L. Buyers and T. Smith, J. Phys. Chem. Solids 29, 105 (1968).

${ }^{17} \mathrm{~B} \cdot \overline{\mathrm{W}}$. Batterman and D. R. Chipman, Phys. Rev. 127690 (1962).

${ }^{18}$ J. C. Phillips, Rev. Mod. Phys. $\underline{42}, 317$ (1970).

\title{
Band Structure of SiGe: Coherent-Potential Approximation*
}

\author{
D. Stroud ${ }^{\dagger}$ and H. Ehrenreich \\ Division of Engineering and Applied Physics, Harvard University, Cambridge, Massachusetts 02138 \\ (Received 4 March 1970)
}

\begin{abstract}
The band structure of SiGe has been calculated using the coherent-potential approximation in conjunction with a realistic but local pseudopotential model. The effects of alloy disorder manifest themselves in complex band energies, each with an imaginary part inversely proportional to the electron lifetime. Spectral functions and the alloy denisty of states are also computed. The damping proves to be small, though it is not always given accurately by loworder-perturbation theory about the virtual crystal. Moreover, within the present local pseudopotential approximation, it affects only $s$ electrons capable of penetrating the ionic cores, since outside the core region the alloy pseudopotential is like that of either limiting pure crystal. The effect of the damping on experimental quantities such as the optical absorption and electrical resistivity is very small.
\end{abstract}

\section{INTRODUCTION}

This paper describes a calculation of the band structure of SiGe, in which we explicitly include the effects of alloy disorder. These manifest themselves in complex band energies with imaginary parts inversely proportional to the electron lifetime. Such a calculation has been made feasible, and potentially useful, by the development of the so-called coherent-potential approximation (CPA) by Soven, ${ }^{1}$ Taylor, ${ }^{2}$ Onodera and Toyozawa, ${ }^{3}$ and Velický,
Kirkpatrick, and Ehrenreich. ${ }^{4}$ The CPA is a method for treating the single-particle properties of substitutionally disordered binary alloys within the framework of multiple-scattering theory. It consists of approximating the configuration-averaged singleparticle alloy Green's function $\langle G\rangle$ by an operator $\bar{G}$ determined by the condition that an electron propagating according to it should undergo, on the average, no scattering at each atomic site. The CPA neglects effects due to the clustering of like atoms 